\title{
Caracterización microestructural y mecánica de aceros de fase dual (ferrita-martensita), obtenidos mediante procesos térmicos y termomecánicos
}

\author{
Microstructural and mechanical characterization of dual phase steels \\ (ferrite-martensite), obtained by thermomechanical processes \\ David Eduardo Castillo Gutiérrez ${ }^{1} \quad$ Irma Inírida Angarita Moncaleano $^{1}$ \\ Rodolfo Rodríguez Baracaldo ${ }^{1 *}$
}

Recibido 28 de diciembre de 2016, aceptado 25 de septiembre de 2017 Received: December 28, 2016 Accepted: September 25, 2017

\begin{abstract}
RESUMEN
En este artículo se discuten las tres rutas diferentes de tratamientos térmicos y termomecánicos para ser utilizados en la obtención de un acero de fase dual (ferrita-martensita), a partir de la modificación microestructural de un acero de bajo carbono AISI/SAE 8620. Se explican las variables de proceso para cada una de las tres rutas empleadas. La caracterización microestructural del material se realizó mediante microscopia óptica y electrónica de barrido y la caracterización mecánica mediante ensayos de dureza, tracción uniaxial, impacto y doblado. Los resultados muestran que el tratamiento de temple intercrítico con enfriamiento en agua, antecedido por un calentamiento con austenización completa con enfriamiento en aire, presentó la mejor combinación de características mecánicas.
\end{abstract}

Palabras clave: Acero de fase dual, tratamientos térmicos, ferrita, martensita, ensayos mecánicos.

\section{ABSTRACT}

Three different thermomechanical treatment and heat treatment process to obtain a dual phase steel (ferritemartensite), from microstructural modification of a low carbon steel AISI / SAE 8620 were evaluated. The processes made for each of these treatments are explained. The microstructural characterization of the material was performed by optical and electron microscopy, and mechanical characterization by hardness, uniaxial tensile, impact and bending test. The results show that intercritical quenching treatment with cooling water preceded by heating above the austenitizing temperature with cooling in air, have the best combination of mechanical properties.

Keywords: Dual phase steel, heat treatment, ferrite, martensite, mechanical tests.

\section{INTRODUCCIÓN}

La selección de materiales es uno de los procesos más importantes en la elaboración de una pieza, puesto que es un factor determinante para los criterios de diseño y fabricación del elemento. La industria automotriz es un ejemplo de la importancia al momento de elegir un material, ya que se buscan partes que cumplan determinados requerimientos como alta resistencia mecánica, bajo peso, alta absorción de energía, entre otros, de tal manera que la pieza sea adecuada para la condiciones propias

\footnotetext{
1 Universidad Nacional de Colombia - Bogotá - Facultad de Ingeniería - Departamento de Ingeniería Mecánica y Mecatrónica Bogotá - Colombia.E-mail: daecastillogu@unal.edu.co; iangaritam@unal.edu.co; rodriguezba@unal.edu.co

* Autor de correspondencia.
} 
de un vehículo y que a su vez se puedan fabricar utilizando las técnicas existentes.

En la actualidad una familia de materiales muy utilizada en la fabricación de vehículos es la de los aceros avanzados de alta resistencia, AHSS (por sus siglas en inglés: Advanced High Strength Steel), este grupo está constituido por los aceros DP (Fase dual), CP (Fase compleja), TRIP (Transformación plástica inducida), MS (Martensíticos) y TWIP (Transformación plástica inducida por maclado) [1]. Los AHSS se caracterizan por tener una mayor resistencia a la fluencia que los HSS (por sus siglas en inglés: High Strength Steel), y en algunos casos una elongación mayor, como se observa en la Figura 1.

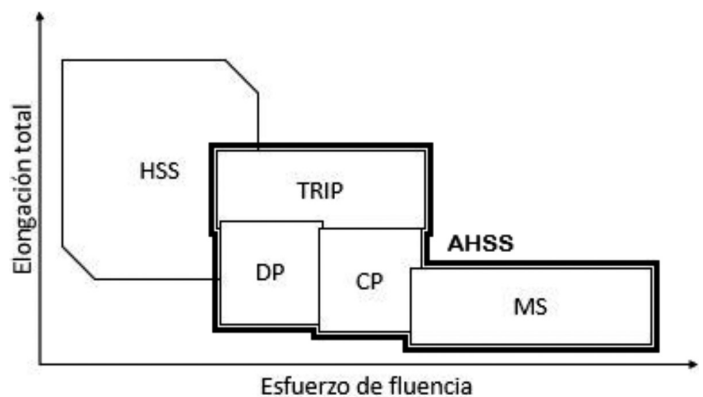

Figura 1. Ductilidad vs. Esfuerzo de fluencia para aceros HSS y AHSS.

Como se mencionó, dentro de los AHSS, se encuentra el acero DP, el que según la ULSAB-AVC (Ultra Light Steel Auto Body - Advance Vehicle Concept), es el material más utilizado para fabricar la mayor cantidad de autopartes, pues hasta un $74 \%$ de las estructura de un vehículo se encuentra elaborada con este acero [2]. La razón de su uso se debe a que el acero de fase dual es capaz de cumplir con los requerimientos propios de una pieza automotriz, teniendo una combinación excepcional entre resistencia y ductilidad [3], debido a que la fase martensítica existente brinda una alta resistencia y la fase ferrítica ofrece una buena ductilidad [4].

En la obtención del acero DP, se utilizan procesos que involucran transformación microestructural por medio de tratamientos térmicos, que en algunos casos están acompañados por altas deformaciones plásticas (termomecánicos). El paso fundamental, en la obtención de un acero DP, consiste en calentar el acero dentro de un rango de temperatura intercrítico definido en los aceros hipoeutectoides, como la región entre las temperaturas críticas $\mathrm{Ac}_{1}$ y $\mathrm{Ac}_{3}$, del diagrama hierro-carbono; donde se producirá la formación de una fase inestable de austenita la que será transformada en martensita [5]. Para la obtención del acero DP, generalmente se utilizan aceros de bajo contenido de carbono y adicional al temple el material puede someterse a recocidos, homogenizaciones o calentamientos con enfriamientos controlados [6]. Los parámetros para definir y evaluar el proceso son: la temperatura intercrítica, el porcentaje de deformación, el que puede llegar a un valor del 95\% [7] y el porcentaje de martensita que se encontrará disperso entre la matriz de ferrita.

El estudio que se plantea a continuación permitió analizar la respuesta de un acero SAE 8620 ante diferentes procesos de trasformación térmica para obtención de aceros de fase dual, de tal manera que se pudo evaluar el efecto que produce la nueva estructura sobre las propiedades del material y se compararon los resultados con estudios previos realizados respecto a este tema.

\section{PROCEDIMIENTO EXPERIMENTAL}

El material de partida para el trabajo fue un acero de bajo carbono AISI-SAE 8620, cuyos porcentajes de composición química se determinaron por espectroscopia de emisión, y se presentan en la Tabla 1.

Tabla 1. Composición química del material de trabajo en estado inicial.

\begin{tabular}{|c|c|c|c|c|c|c|}
\hline Elemento & $\mathbf{C}$ & $\mathbf{N i}$ & $\mathbf{C r}$ & $\mathbf{M n}$ & $\mathbf{M o}$ & $\mathbf{F e}$ \\
\hline Masa (\%) & 0,2 & 0,7 & 0,6 & 0,9 & 0,25 & Bal. \\
\hline
\end{tabular}

Un esquema de los tratamientos realizados se representa en la Figura 2. El tratamiento 1 consistió en un austenizado inicial a $915^{\circ} \mathrm{C}$ durante 30 minutos $\mathrm{y}$ enfriamiento en aire (normalizado), seguido por un calentamiento a una temperatura intercrítica de $780{ }^{\circ} \mathrm{C}$ por 30 minutos, finalizando con un temple en agua.

El tratamiento 2 consistió en un calentamiento a $915{ }^{\circ} \mathrm{C}$ por 30 minutos, con un enfriamiento 
a.

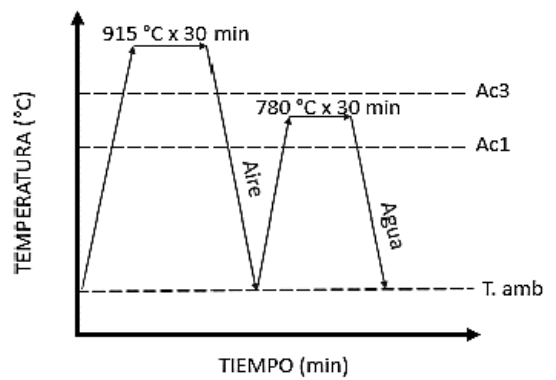

b.

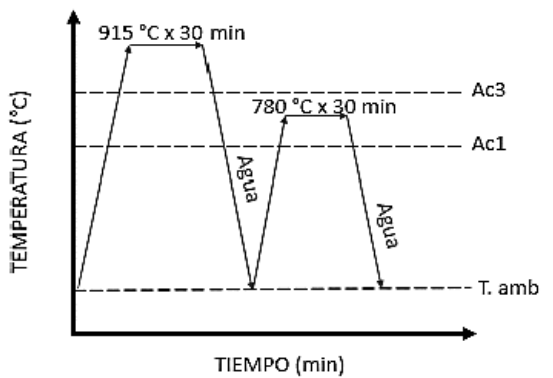

c.

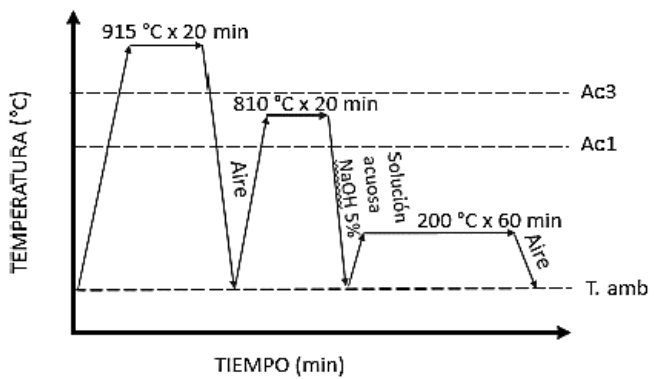

Figura 2. Esquemas de proceso a) Tratamiento 1, b) Tratamiento 2, c) Tratamiento 3.

posterior en agua, después el material es calentado en el rango intercrítico por 30 minutos a $780{ }^{\circ} \mathrm{C}$ y enfriado en agua nuevamente.

El tratamiento 3 consistió en un proceso termomecánico después que el material se normalizó desde una temperatura de austenización de $915^{\circ} \mathrm{C}$ durante 20 minutos, luego se llevó a una temperatura intercrítica de $810^{\circ} \mathrm{C}$ durante 20 minutos, posteriormente se aplicó una deformación del $10 \%$ en caliente, seguido de un temple en solución de $\mathrm{NaOH}$ al 5\%; el tratamiento terminó con un revenido por 1 hora a $200{ }^{\circ} \mathrm{C}$. Los tiempos y temperaturas usadas para el proceso de estabilización de la austenita están basados en estudios previos sobre el tema, [8], [9], [10].

Posterior a los tratamientos térmicos y termomecánicos fueron realizados ensayos de laboratorio con el fin de caracterizar la microestructura y las propiedades mecánicas del material. En la caracterización microestructural se realizaron pruebas de composición química, microscopia óptica, microscopia electrónica de barrido (SEM, por sus siglas en inglés Scanning Electron Microscopy) y microdureza. Se utilizaron muestras en forma de disco de $19 \mathrm{~mm}$ de diámetro y espesor de $6 \mathrm{~mm}$ cortados a la mitad para poder hacer una observación de las secciones transversales y longitudinales del material. Las muestras fueron encapsuladas en resina epóxica, desbastadas con lijas de grano grueso, medio y fino, y pulidas con paño usando alúmina como material abrasivo. El ataque químico se hizo con Nital en una concentración del $2 \%$. La observación de la microestructura se efectuó en microscopio óptico, marca Olympus X30, determinando el tamaño de grano y las fases presentes. La dureza de las fases fue tomada en un microdurómetro marca Leco 402 con un mínimo de 6 indentaciones, carga de $200 \mathrm{~g}$ y tiempo de 10 segundos. Finalmente, se empleó un microscopio electrónico FEI, modelo Quanta 200 para observar en detalle las fases microestructurales y la superficie de fractura de los ensayos de impacto y tracción.

La caracterización de las propiedades mecánicas y tecnológicas del material se realizó por medio de ensayos de dureza, tracción uniaxial, impacto y flexión. Para la dureza el equipo utilizado fue un durómetro análogo Wolpert, las mediciones se efectuaron en escala Rockwell B. Se hizo un perfil de dureza desde el centro de la probeta (eje axial de la pieza), hasta el extremo de la misma tomando mediciones cada $2 \mathrm{~mm}$. Todas las mediciones presentaron un margen de error de $+/-5 \%$ por condiciones del equipo.

Los ensayos de tracción uniaxial fueron realizados con probetas de longitud calibrada de $50 \mathrm{~mm}$ y diámetro de cuello de 12,7 mm según la norma ASTM E-8 [11]. El equipo utilizado fue una máquina universal de ensayos Shimadzu UH_X con control de desplazamiento a una velocidad del cabezal de $5 \mathrm{~mm} / \mathrm{min}$. La superficie de fractura resultante fue analizada mediante microscopia electrónica de barrido.

Para la prueba de flexión se fabricaron probetas con dimensiones 4 × 6 × $57 \mathrm{~mm}$ de acuerdo con la norma ASTM E-290 [12]. Se optó por realizar dobleces en $\mathrm{V}$, utilizando un punzón a $30^{\circ}$ y una matriz a $80^{\circ}$; la carga aplicada fue de $45 \mathrm{~kg} / \mathrm{mm}^{2}$. Para garantizar una 
interfaz punzón-pieza-matriz lubricada se recubrieron las piezas con película de polipropileno y el punzón fue cubierto en su punta con grasa de jabón de litio. En cada prueba se tomaron fotografías a las piezas cuando fueron sometidas a carga y cuando esta era retirada, posteriormente haciendo uso de software $\mathrm{CAD}$, se midió el ángulo durante la aplicación $\left(\mathrm{A}_{\text {carga }}\right)$ y después de retirada la carga $\left(\mathrm{A}_{\text {descarga }}\right)$ para ver el efecto que este tenía sobre la pieza. Con los valores de ángulo de carga y descarga se calculó la relación $K$ explicada en la ecuación (1) [13], como criterio para evaluar la conformabilidad.

$$
K=\frac{A_{\text {carga }}}{A_{\text {descarga }}}
$$

Los ensayos de impacto Charpy, se realizaron con probetas de $5 \times 5 \times 55 \mathrm{~mm}$, con una profundidad de entalla de $1 \mathrm{~mm}$ y ángulo de $45^{\circ}$ según la norma ATSM E-23 [14]. Las muestras fueron tratadas térmicamente y posteriormente mecanizadas. Se empleó una máquina de impacto marca WPM con una sensibilidad de $1 \mathrm{~N} \cdot \mathrm{m}$.

\section{RESULTADOS}

\section{Caracterización microestructural}

En las Figuras 3 (a) a (d) se presentan las microestructuras del acero en estado inicial y de cada tratamiento realizado obtenidas con microscopio óptico. La Figura 4 (a) a (d) muestra las microestructuras, tomadas mediante SEM.

En las microestructuras tomadas con microscopio óptico en los aceros tratados térmicamente, se observa la presencia de las dos fases, correspondientes a la martensita (regiones oscuras), y a la ferrita (regiones claras). No se visualizan diferencias notorias entre el tratamiento 1 (Figura $3 \mathrm{~b}$ ) y el tratamiento 2 (Figura 3c), mientras que el tratamiento 3 (Figura 3d) presenta granos más asimétricos y alargados.
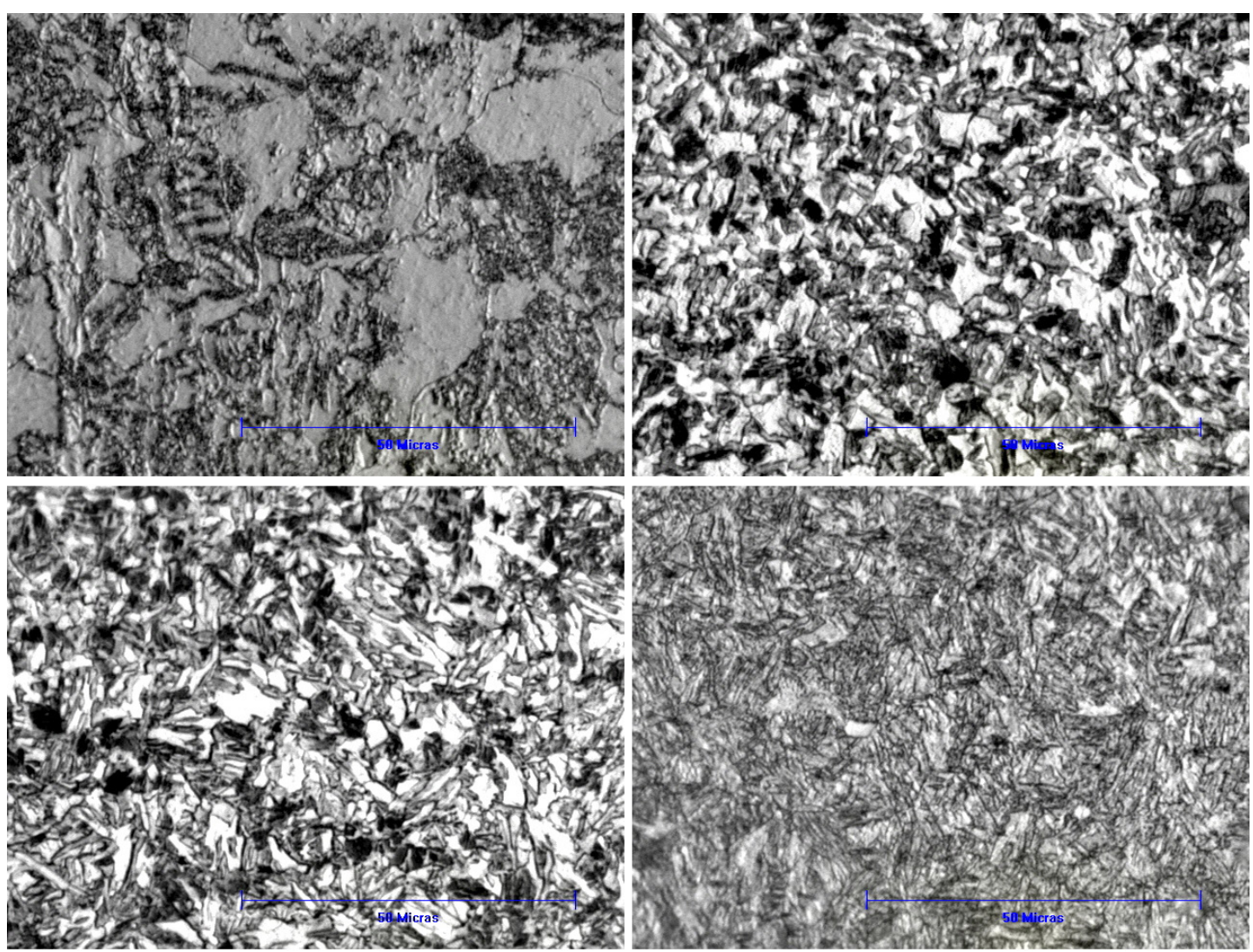

Figura 3. Micrografías de la microestructura de los aceros: a) Estado inicial, b) Tratamiento 1, c) Tratamiento 2, d) Tratamiento 3. 

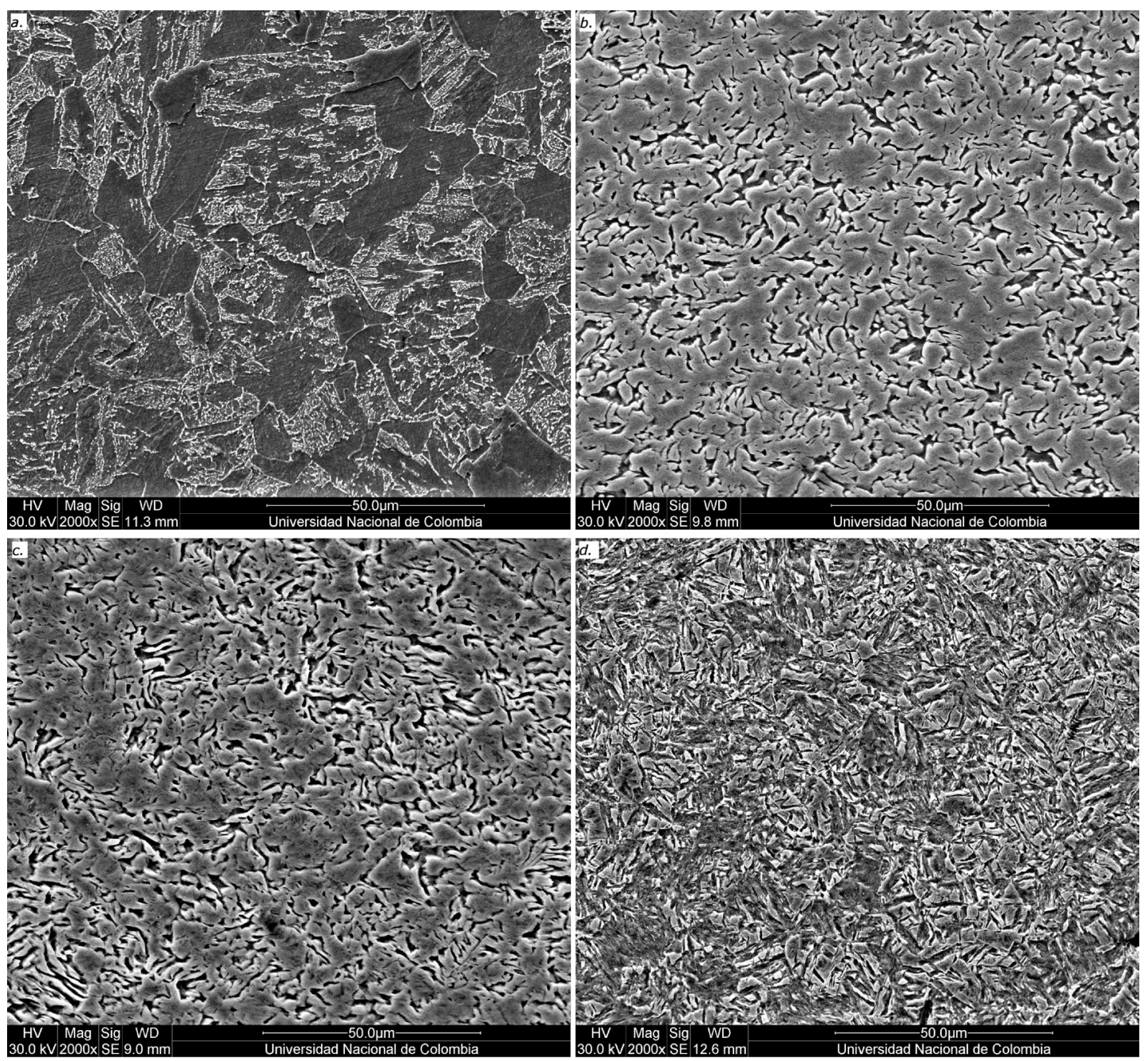

Figura 4. Fotografías de la microestructura en SEM de los aceros: a) Estado in icial, b) Tratamiento 1, c) Tratamiento 2, d) Tratamiento 3 .

En las microestructuras obtenidas mediante SEM de la Figura 4, se ve la diferencia entre los aceros tratados térmicamente y el acero en estado inicial (Figura 4a). Los que fueron sometidos a procesos de transformación presentan estructuras de grano acicular propias de la martensita. También se observa una menor cantidad de martensita dispersa sobre la matriz de ferrita en el tratamiento 1 (Figura 4b), con respecto al tratamiento 2 (Figura $4 \mathrm{c}$ ) y 3 (Figura 4d), en el caso de este último se presenta un alargamiento en una dirección preferencial dada por la deformación.

El tamaño de grano medido y los resultados de la prueba de microdureza, se encuentra en la Tabla 2.
Se empleó el método de la intercepción en mínimo cinco micrografías de cada tipo de material. El tamaño de grano en el tratamiento 1 , es mayor que en el tratamiento 2 y 3 ; la causa más viable es el uso de medios de enfriamientos más severos para estos dos procesos, durante las diferentes etapas. Los tamaños de grano medidos son menores respecto al acero de fase dual obtenido por A. Monsalve [6], posiblemente por la disminución controlada de la temperatura desde la zona de austenización que realizaron en dicha investigación, lo que facilita la recuperación del material.

Debido a las características del proceso el material al que se le realizó el tratamiento 1, presentó menor 
Tabla 2. Resultados promedio de microdureza y tamaño de grano (desviación estándar se muestra entre paréntesis).

\begin{tabular}{|c|c|c|}
\hline Material & $\begin{array}{c}\text { Microdureza } \\
(\mathbf{H V})_{\mathbf{0 , 2}}\end{array}$ & $\begin{array}{c}\text { Tamaño de } \\
\text { grano }(\boldsymbol{\mu m})\end{array}$ \\
\hline Estado inicial & $368,5(17,2)$ & $23,7(1,6)$ \\
\hline Tratamiento 1 & $442,6(18,0)$ & $11,4(2)$ \\
\hline Tratamiento 2 & $485,8(14,7)$ & $9,5(2)$ \\
\hline Tratamiento 3 & $465,8(8,2)$ & $9,7(2)$ \\
\hline
\end{tabular}

dureza, por el efecto de la recuperación del grano durante el normalizado. En el tratamiento 2 se puede ver el efecto del temple desde la temperatura de austenización e intercrítica, donde se produce la mayor densidad de dislocaciones generando una elevada dureza. El tratamiento 3 tuvo valores intermedios de microdureza, debido al proceso de revenido posterior al temple el que genera una microestructura con menor dureza, a pesar de haber sido templado el material en $\mathrm{NaOH}$. Pruebas de microdureza como las de E. Cárdenas [5], muestran resultados de microdureza mayores, posiblemente debido a que los aceros de esta investigación presentan cantidades de carbono menores a los evaluados por Cárdenas.

\section{Caracterización mecánica}

La Figura 5 presenta el perfil de dureza del material en función de la distancia, donde el 0 corresponde al centro del disco utilizado.

En comparación con el material en estado inicial, todos los aceros tratados térmicamente presentan

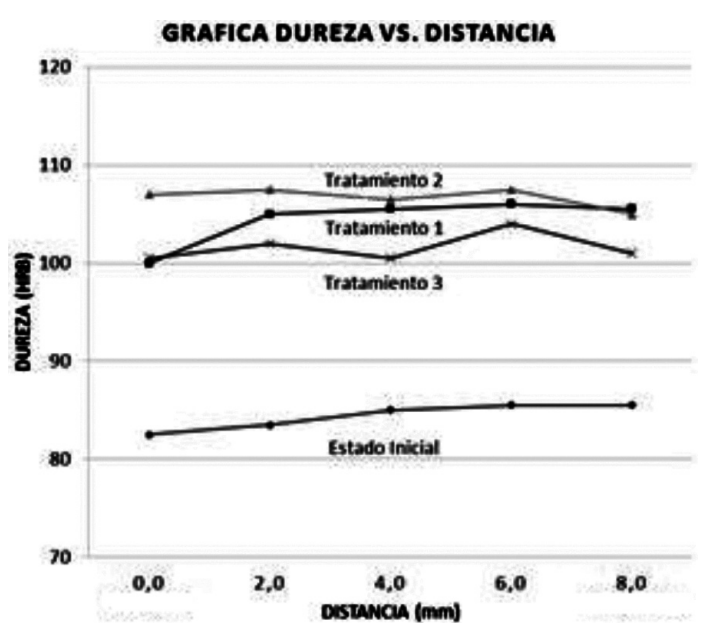

Figura 5. Gráfica dureza vs. distancia. un incremento en su dureza. Para el tratamiento 1 se encuentra una disminución de la magnitud en el centro del material; este fenómeno sugiere una recuperación por las condiciones del proceso de normalizado previo al temple intercrítico, formando una ferrita de mayor tamaño y simetría en las dimenciones, lo que no sucede en los tratamientos 2 y 3 donde la dureza tuvo valores constantes en toda la sección. En trabajo de N. Saeidi [15], los aceros duales muestran una mayor dureza, una de las posibles causas puede ser una mayor proporción de la combinación de aleantes níquelcromo, en el material inicial, teniendo en cuenta que la presencia de estos elementos favorece el endurecimiento del acero.

La Figura 6 presenta la curvas de esfuerzo vs. deformación que se obtuvieron de las muestras por medio del ensayo de tracción uniaxial.

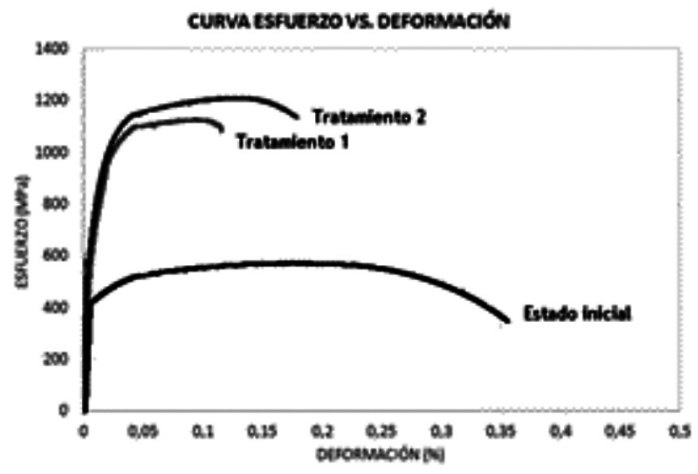

Figura 6. Gráfica esfuerzo vs. deformación.

Los valores de las propiedades extraídas de las curvas mostradas en la Figura 6 se resumen en la Tabla 3. Debido a las limitaciones geométricas para realizar el tratamiento 3 , estas probetas no fueran sometidas a los ensayos de tracción; por tanto el esfuerzo máximo fue estimado a partir de los valores de dureza, considerando la relación de Tabor (Esfuerzo máximo = 3,4 dureza Brinell) [16]

La resistencia máxima y de fluencia del material aumentan en las muestras tratadas con respecto a la del acero en estado inicial, debido a la presencia de la fase martensitica y al aumento de la densidad de dislocaciones que se forman durante el proceso de transformación de austenita en martensita, como fue señalado por A. Kumar [17]. En los valores de 
Tabla 3. Valores de propiedades de tracción.

\begin{tabular}{|l|c|c|c|}
\hline Material & $\begin{array}{c}\text { Esfuerzo } \\
\text { máximo } \\
\text { (MPa) }\end{array}$ & $\begin{array}{c}\text { Esfuerzo } \\
\text { de fluencia } \\
\text { (MPa) }\end{array}$ & $\begin{array}{c}\text { Deformación } \\
(\boldsymbol{\%})\end{array}$ \\
\hline Estado inicial & 588,26 & 412,48 & 36,94 \\
\hline Tratamiento 1 & $1.163,0$ & 523,68 & 9,92 \\
\hline Tratamiento 2 & $1.252,96$ & 586,24 & 14,96 \\
\hline Tratamiento 3 & 836,31 & - & - \\
\hline
\end{tabular}

esfuerzo máximo es donde ocurren las diferencias mayores entre los aceros, lo que refleja diferente capacidad de endurecimiento en la región plástica del material. Los tratamientos 1 y 2 , donde no hay deformación termomecánica, los resultados del presente estudio tienen una correspondencia con lo hecho por U. Liedl [4] y N. Saeidi [15], para los valores de esfuerzo máximo y de fluencia con respecto al porcentaje de carbono del acero inicial. En el caso del trabajo de U. Liedl [4], con menor cantidad de carbono reporta una menor resistencia mecánica. Para el caso de N. Saeidi [15] al tener mayor cantidad de carbono, se obtiene un mayor esfuerzo máximo y de fluencia. En el tratamiento 3 el valor calculado de esfuerzo máximo es similar con el reportado por E. Ahmad [18], quien sometió el material a un proceso de deformación en caliente.

En la Figura 7 se pueden observar las probetas falladas a tensión. El acero en estado inicial presentó un encuellamiento en la longitud calibrada, propio de una falla dúctil. Por otra parte, los aceros tratados térmicamente muestran una zona de rotura frágil, debido al aumento de la cantidad de martensita en la microestructura del material, a causa del proceso

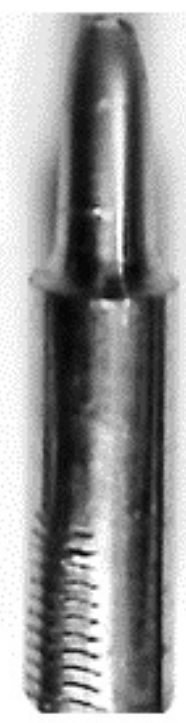

a.

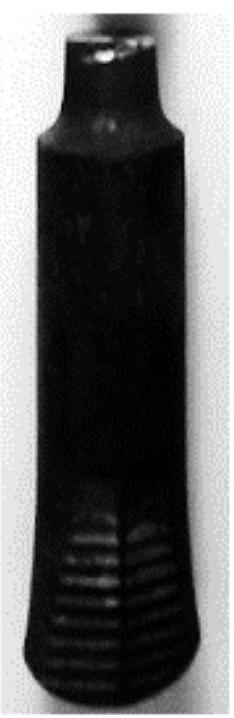

b.

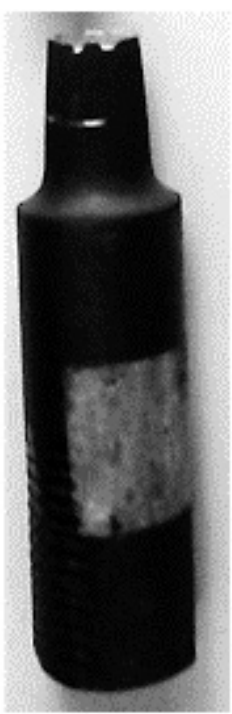

C.
Figura 7. Probetas falladas por ensayo de tracción uniaxial: a) Estado inicial, b) Tratamiento 1, c) Tratamiento 2 .

de trasformación de fase generado mediante los tratamientos térmicos, lo que incrementó su dureza.

Las fotografías obtenidas por SEM correspondientes a la superficie de falla de las probetas sometidas al ensayo de tracción uniaxial se presentan en la Figura 8.

El acero en estado inicial (Figura 8a) tiene una falla dúctil con una gran cantidad de microvacíos sobre la superficie. El tratamiento 1 (Figura 8b) muestra una fractura intergranular (desprendimiento del grano), acompañadas por pequeños sectores de clivaje.

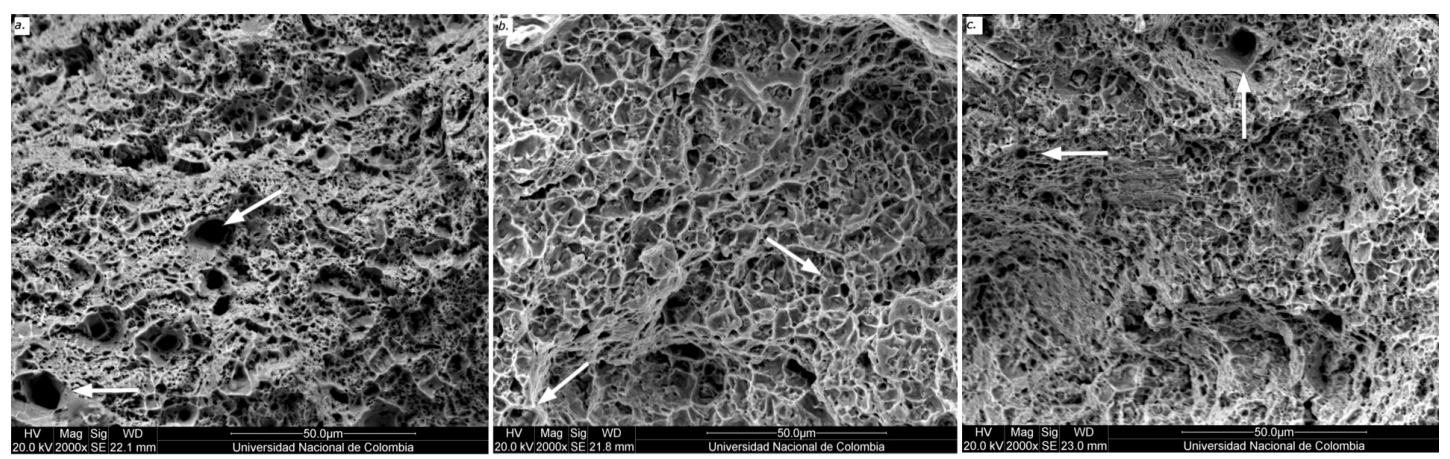

Figura 8. Fotografías SEM de la superficie de falla en tracción uniaxial: a) Estado inicial, b) Tratamiento 1, c) Tratamiento 2 . 
Para el tratamiento 2 (Figura 8c) se presentó de una manera similar falla intergranular, pero con una mayor presencia de microvacíos, lo que indica una mejor ductilidad respecto al tratamiento 1 , así como lo reflejan las curvas de esfuerzo vs. deformación de la Figura 6.

La Tabla 4 presenta los resultados del ensayo de flexion para las muestras analizadas en cada tratamiento. La conformabilidad del material será más alta a medida que el factor $\mathrm{K}$ sea más cercano a 1 , pues esto indicará que el material mantiene su forma después de retirada la carga.

Tabla 4. Resultados promedio para ensayo de flexión, con desviación estándar.

\begin{tabular}{|c|c|c|c|}
\hline \multicolumn{1}{|c|}{ Material } & $\mathbf{A}_{\text {carga }}$ & $\mathbf{A}_{\text {descarga }}$ & Factor K \\
\hline \multirow{2}{*}{ Estado inicial } & $\begin{array}{c}89,67 \\
(0,58)\end{array}$ & $\begin{array}{c}89,72 \\
(1,50)\end{array}$ & $\begin{array}{c}0,9996 \\
(0,0134)\end{array}$ \\
\hline \multirow{2}{*}{ Tratamiento 1 } & $\begin{array}{c}89,80 \\
(0,81)\end{array}$ & $\begin{array}{c}90,69 \\
(0,73)\end{array}$ & $\begin{array}{c}0,9996 \\
(0,0010)\end{array}$ \\
\hline \multirow{2}{*}{ Tratamiento 2 } & $\begin{array}{c}89,58 \\
(1,02)\end{array}$ & $\begin{array}{c}93,78 \\
(0,45)\end{array}$ & $\begin{array}{c}0,9552 \\
(0,0076)\end{array}$ \\
\hline \multirow{2}{*}{ Tratamiento 3 } & $\begin{array}{c}88,48 \\
(0,79)\end{array}$ & $\begin{array}{c}92,02 \\
(1,29)\end{array}$ & $\begin{array}{c}0,9616 \\
(0,0086)\end{array}$ \\
\hline
\end{tabular}

El tratamiento 1 y el tratamiento 2 presentan mejor relación K que el tratamiento 3. Sin embargo, para las probetas del tratamiento 1 se generó una fractura parcial que invalida los resultados del factor de recuperación para estas muestras.

En el tratamiento 2 las fases de martensita y ferrita permiten una combinación adecuada de resistencia y ductilidad que favorece la deformación del material sin que exista recuperación elástica excesiva o fractura de la muestra. En el caso del tratamiento 3 el valor de $\mathrm{K}$ es menor, con un comportamiento muy cercano al del material en estado inicial; una razón de este comportamiento es la mayor cantidad de martensita dispersa sobre la matriz de ferrita, y la presencia de un alargamiento preferencial de grano dado por la deformación.

La Figura 9 muestra los valores de energía absorbida promedio obtenida mediante el ensayo Charpy. Los resultados se clasifican en dos grupos, altos y bajos valores de energía absorbida; el primer caso corresponde al tratamiento $1 \mathrm{y}$ el acero en estado inicial, mientras que el segundo agrupa a los tratamientos 2 y 3 . Este comportamiento se entiende a partir de los resultados de la prueba dureza en el centro de la muestra de trabajo (distancia de $0 \mathrm{~mm}$ en la Figura 5), existiendo una relación inversa con la energía que puede absorber la pieza. Lo anterior permite inferir que el tratamiento 1 por la formación y disposición de la martensita dada tiene una buena resistencia en la periferia del material y por causa del crecimiento del grano una mayor ductilidad en el núcleo permitiendo que se tenga un material de mayor tenacidad en su conjunto [19].

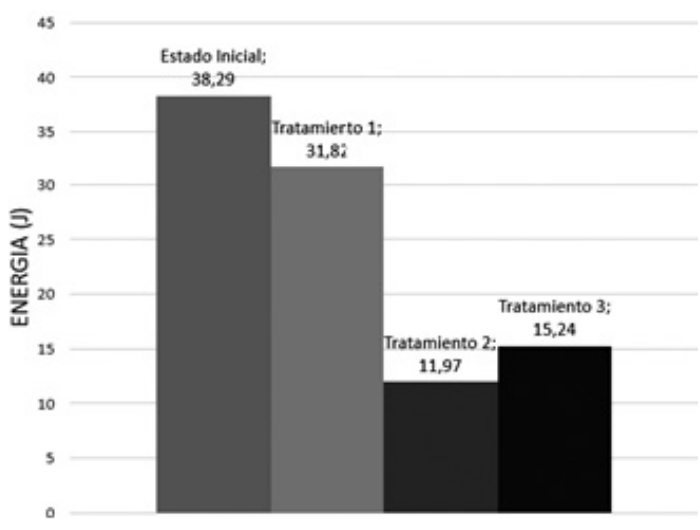

Figura 9. Resultado promedio para ensayo Charpy de energía de impacto, con desviación estándar.

\section{CONCLUSIONES}

Por medio de la caracterización estructural y mecánica de los materiales resultantes en función de los tratamientos realizados se encontró que en todos los casos existe un comportamiento acorde al de un acero de fase dual constituido por una microestructura de ferrita-martensita.

El tratamiento $1 \mathrm{y}$ tratamiento 2 , donde no se involucran deformaciones en caliente, presentan los mejores resultados brindando aceros con buenas características de resistencia mecánica y conformabilidad. Adicionalmente, el material sometido al tratamiento 1 tiene una mayor capacidad de absorber energía de impacto.

En el caso del tratamiento 1 se puede observar que durante la primera etapa de normalizado, el material tiene la posibilidad de una recuperación 
microestructural, homogenizando el tamaño de grano y eliminación de heterogeneidades producidas de la fabricación, esto permite tener un acero que para la fase posterior de temple intercrítico se encuentre mejor acondicionado para la transformación estructural.

\section{AGRADECIMIENTOS}

Los autores agradecen a la Vicerrectoría de Investigación de la Universidad Nacional de Colombia por el apoyo para el desarrollo del presente proyecto.

\section{REFERENCIAS}

[1] M. Ruiz Andrés. "Caracterización Microestructural, Micromecánica y Tribológica de Aceros Dual Phase de Alta Resistencia Sometidos a Procesos de Perfilado En Frío". Tesis para optar al grado de Magíster. Universidad Complutense de Madrid, Facultad de Ciencias Físicas, Departamento de Física de Materiales. Madrid, España. 2012.

[2] J. Madias. "Avances en la producción y aplicación de aceros bifásicos", pp. 2-10. Junio 2014. Fecha de consulta: 18 de febrero de 2015. URL: https://www.researchgate. net/publication/262729018_Avances_en_ la_produccion_y_aplicacion_de_aceros_ bifasicos

[3] P. Tsipoudiris. "Mechanical properties of Dual-phase steels". Tesis para optar al grado de doctor. Technische Universitat Munchen. Materialprüfamt für den Mashinenbau. Munich, Alemania. 2006.

[4] U. Liedl, S. Traint, E.A. Werner. "An unexpected feature of the stress-strain diagram of dual-phase steel". Computational Materials Science. Vol. 25 No 1, pp. 122-128. Septiembre 2002. ISSN: 0927-0256 DOI: 10.1016/S0927-0256(02)00256-2.

[5] E. Cárdenas, C. Rodríguez, F.J. Belzunce y C. Betegón. "Estudio de las propiedades mecánicas de aceros de doble fase mediante el ensayo miniatura de punzonado". Anales de la Mecánicas a la Fractura 26. Secretaria del Grupo Español de Fractura. Santander, España. Vol. 1, pp. 166-171. 2009. ISSN: 0213-3725.

[6] A. Monsalve G., A. Artigas A., F. Castro C., R. Colás e Y. Houbaert. "Caracterización de aceros dual-phase obtenidos por laminación en caliente". Revista de Metalurgia. Vol. $47 \mathrm{~N}^{\circ} 1$, pp. 5-14. Enero-febrero 2011. ISSN: 00348570. DOI: 10.3989/revmetalmadrid.0914

[7] S. Sun and M. Pugh. "Properties of thermomechanically processed dual-phase steels containing fibrous martensite". Materials Science and Engineering A. Vol. $353 \mathrm{~N}^{\circ} 1-2$, pp. 298-308. Septiembre 2002. ISSN: 0921-5093. DOI: 10.1016/ S0921-5093(01)01942-6.

[8] Raúl E. Riveros T. "Estudio de las propiedades del acero 1045 con microestructura de ferrita y martensita revenida (Dual-Phase)". Tesis para optar al grado de ingeniero. Universidad Nacional de Colombia. Facultad de Ingeniería. Departamento de Ingeniería Mecánica. Bogotá D.C., Colombia. 1999.

[9] E. Ahmad, T. Manzoor, N. Hussain and N.K. Qazi. "Effect of thermomechanical processing on hardenability and tensile fracture of dual-phase steel". Materials and Design. Vol. 29 No $^{\circ}$ 2, pp. 450-457. Enero 2008. ISSN: 0264-1275 DOI: 10.1016/j. matdes.2006.12.022.

[10] R. Rodríguez Baracaldo, J.M. Arroyo Osorio y Y. Parra Rodríguez. "Influencia del proceso de revenido en el comportamiento mecánico de un acero de fase dual de uso industrial automotriz". Ingeniare. Revista Chilena de Ingeniería. Vol. $24 \mathrm{~N}^{\circ} 1$, pp. 94-101. Enero 2016. ISSN: 0718-3305 DOI: $10.4067 /$ S0718-33052016000100009.

[11] ASTM International. "ASTM E8 / E8M-13: Standard Test Methods for Tension Testing of Metallic Materials" West Conshohocken, PA, Estados Unidos. 2013.

[12] ASTM International. "ASTM E290-13: Standard Test Methods for Bend Testing of Material for Ductility". West Conshohocken, PA, Estados Unidos. 2013.

[13] A. Ivanišević, M. Milutinović, B. Štrbac and P. Skakun. "Stress state and spring back in v-bending operations". International Journal for Technology of Plasticity. Volumen 39, Número 2, pp. 157-168. Julio 2013. Fecha de consulta: 30 de marzo de 2015 ISSN: 0749-6419. URL: http://www.dpm.ftn.uns. ac.rs/JTP/Download/2013/2/Article5.pdf

[14] ASTM International. "ASTM E23-12C: Standard Test Methods for Notched Bar 
Impact Testing of Metallic Materials". West Conshohocken, PA, Estados Unidos. 2012.

[15] N. Saeidi, A. Ekrami. "Comparison of mechanical properties of martensite/ ferrite and bainite/ferrite dual phase 4340 steels". Materials Science and Engineering A. Vol. $523 \mathrm{~N}^{\circ} 1$, pp. 125-129. Octubre 2009. ISSN: 0921-5093. DOI: 10.1016/j. msea.2009.06.057.

[16] G.E. Dieter. "Mechanical Metallurgy". Editorial McGraw-Hill Book Company. Primera edición. New York, Estados Unidos, pp. 262-269. 1961. ISBN: 07-016890-3.

[17] A. Kumar, S.B. Singh and K.K. Ray. Materials "Influence of Bainite/Martensite-Content on the Tensile Properties of Low Carbon
Dual-Phase Steels". Science and Engineering A. Vol. $474 \mathrm{~N}^{\circ}$ 1-2, pp. 270-282. Febrero 2008. ISSN: 0921-5093. DOI: 10.1016/j. msea.2007.05.007.

[18] E. Ahmad, T. Manzoor, N. Hussain and N.K. Qazi. "Thermomechanical processing in the intercritical region and tensile properties of dual-phase steel". Materials Science and Engineering A. Vol. $508 \mathrm{~N}^{\circ}$ 1, pp. 259265. Mayo 2009. ISSN: 0921-5093. DOI: 10.1016/j.msea.2009.01.056.

[19] M.A. Meyers and K.K. Chawla. "Mechanical Behavior of Materials". Editorial Cambridge University Press. Segunda edición. Cambridge Reino Unido, pp. 526. 2009. ISBN: 978-0-521-86675-0. 